\title{
Chemical-Equilibrium Analysis with Adjoint Derivatives for Propulsion Cycle Analysis
}

\author{
Justin Gray,, Jeffrey Chin,, \pm Tristan Hearn,, \pm Eric Hendricks,, \pm and Thomas Lavelle $₫$ \\ NASA John H. Glenn Research Center, Cleveland, Ohio 44139 \\ and \\ Joaquim R. R. A. Martins $\underline{\S}$ \\ University of Michigan, Ann Arbor, Michigan 48109 \\ DOI: $10.2514 / 1 . B 36215$
}

\begin{abstract}
The design optimization of aircraft engines considering their integration with the airframe has been limited by challenges with existing propulsion modeling tools. Gradient-based optimization with derivatives computed using adjoint methods has been successful in solving aerodynamic and structural shape optimization problems but has not yet been applied to coupled propulsion-airframe optimization, partly because existing tools lack analytic derivative computation. As a step toward obtaining a full cycle analysis with efficient analytic derivative computation, a new chemical-equilibrium thermodynamics solver is developed for propulsion applications. This solver provides a continuous formulation that enables analytic derivative computation using a coupled adjoint approach. The results from this solver are verified against a well-established chemical-equilibrium code. The analytic derivatives are also verified by comparing them with finite-difference approximations. The performance of the analytic derivative computations is tested using two optimizations: combustion temperature maximization with respect to equivalence ratio, and combustion temperature maximization with respect to air pressure. The results show clear speed and numerical stability benefits when comparing the proposed method against finite-difference approximations. It is now possible to use this new solver as the foundation for further development of a complete propulsion analysis for integrated propulsion-airframe design optimization.
\end{abstract}

\section{Nomenclature}

$a_{i j}=$ stoichiometric constant for the $i$ th element in the $i$ th species

$b=$ moles of an element summed across all species in a gas

$b^{\circ}=$ moles of an element summed across all species in a gas at the initial composition

$C_{P}=$ specific heat at constant pressure

$C_{V}=$ specific heat at constant volume

$G=$ Lagrangian of the Gibbs energy minimization

$g=$ Gibbs energy

$H^{\circ}=$ enthalpy of a species as a function of $T$

$h=$ specific enthalpy

$h_{0}=$ input specified enthalpy

$N_{e}=$ total number of elements

$N_{s}=$ total number of chemical species

$n=$ concentration of chemical species, $\mathrm{kg} \cdot \mathrm{mol} / \mathrm{kg} \cdot$ mixture

$P=$ pressure

$P_{a}=$ pressure at standard conditions (1.01325 bar)

$R=$ universal gas constant

$\mathcal{R}=$ residual function

$S=$ entropy

$S_{0} \quad=$ input specified entropy

Presented as Paper 2016-0669 at the 57th AIAA/ASCE/AHS/ASC Structures, Structural Dynamics, and Materials Conference, San Diego, CA, 4-8 January 2016; received 17 February 2016; revision received 7 October 2016; accepted for publication 20 October 2016; published online 30 January 2017. This material is declared a work of the U.S. Government and is not subject to copyright protection in the United States. All requests for copying and permission to reprint should be submitted to CCC at www.copyright.com; employ the ISSN 0748-4658 (print) or 1533-3876 (online) to initiate your request. See also AIAA Rights and Permissions www.aiaa.org/randp.

*Aerospace Engineer, PSA Branch, 21000 Brookpark Rd., MS 5-11; also Doctoral Candidate, University of Michigan, Department of Aerospace Engineering, Ann Arbor, MI 48109. Member AIAA.

${ }^{\dagger}$ Aerospace Engineer, PSA Branch, 21000 Brookpark Rd., MS 5-11. Member AIAA.

¥Aerospace Engineer, PSA Branch, 21000 Brookpark Rd., MS 5-11.

${ }^{\S}$ Professor, Department of Aerospace Engineering. Associate Fellow AIAA.
$S^{\circ} \quad=\quad$ entropy of a species as a function of $T$

$T=$ temperature

$U=$ vector of state variables in the chemical-equilibrium equations

$\gamma=$ ratio of specific heats $\left(C_{P} / C_{V}\right)$

$\lambda=$ Lagrange multipliers

$\mu \quad=$ chemical potential energy

$\pi=$ modified Lagrange multipliers

$\rho \quad=$ density

$\phi \quad=$ air-fuel equivalence ratio

\section{Subscripts}

$i=$ quantity for the $i$ th element

$j \quad=\quad$ quantity for the $j$ th chemical species

$k=$ alternate subscript for the $k$ th chemical species

\section{Introduction}

$\mathbf{N}$ EW aircraft concepts with a high degree of propulsion-airframe integration have been proposed to dramatically reduce fuel burn, emissions, and noise. These concepts (such as overwing nacelles $[1,2]$ and engine installations designed for boundary-layer ingestion (BLI) [3-6]) couple the thermodynamic performance of the propulsion system with the aerodynamic performance of the airframe. This requires coupling the aerodynamic and propulsion analysis tools for aircraft design and motivates the application of multidisciplinary design analysis and optimization (MDAO) to navigate the potentially large design space [7]. Drela [8] underscored the importance of capturing this coupling in the D8 aircraft concept, and Welstead and Felder [9] made the same point for the Single Aisle Turboelectric Aircraft Concept-Aft Boundary Layer Ingestion (Starc-ABL) concept.

Applying MDAO requires a set of analysis tools well suited to optimization. This means that the tools must be numerically stable, be capable of returning physically meaningful results even when starting with poor designs, and be computationally efficient. In cases where gradient-based optimization is used, it is highly desirable that the analysis tools can efficiently and accurately compute derivatives, and that the formulation of the analysis leads to smooth and 
differentiable functions. Computing the derivatives of a function of interest is always possible by using finite-difference approximations. However, such an approach is not efficient because the cost of computing the complete gradient is proportional to the number of variables. In addition, this method is subject to errors due to subtractive cancellation [10]. Analytic methods, where the analysis is linearized, are much more accurate and generally more efficient [11]. Two main forms of analytic methods exist: the direct method and the adjoint method. The computational cost of the direct method is proportional to the number of design variables and independent of the number of functions of interest, whereas the cost of the adjoint method is proportional to the number of functions of interest and independent of the number of design variables.

Applying MDAO to propulsion-airframe integration problems requires coupling viscous computational fluid dynamics (CFD) to propulsion analysis. When using CFD-based shape optimization, the most common technique is to use gradient-based optimization with the gradients computed via the adjoint method. The reason for this choice is that there are few functions of interest (usually drag, lift, and moment coefficients), but a large number of design variables are required to parametrize three-dimensional shapes. Many CFD tools that compute adjoint derivatives are already available, such as FUN3D [12], SU2 [13], elsA [14], and ADflow [15-18]. The adjoint method, in conjunction with gradient-based optimization, has been used in many studies of aerodynamic shape optimization, as demonstrated by the series of benchmarks developed by the AIAA Aerodynamic Design Optimization Discussion Group [14,17,19-21]. However, propulsion analysis tools are not as well developed in this regard, and many existing applications still rely on gradient-free methods, which cannot handle large numbers of design variables.

To date, gradient-free methods have been successful because the number of design variables required to formulate the problem has been kept relatively small, but the integration of propulsion analysis with viscous CFD requires a large number of design variables, which makes gradient-based optimization a requirement [22]. Sandhi et al. [23] identified many different options in their survey of propulsion modeling tools, but none computed analytic derivatives. Of the tools identified, numerical propulsion system simulation (NPSS) [24] is the most recently developed and is widely used. Because NPSS does not compute analytic derivatives, the finite-difference method is used to approximate derivatives. The finite-difference derivatives from NPSS could, in theory, be combined with the adjoint derivatives from the CFD tool to form a semianalytic coupled derivative that would enable gradient-based optimization.

Although the semianalytic approach would allow integrated propulsion-airframe optimization with existing tools, such a solution is far from ideal. Finite-difference derivative approximations from NPSS result in numerical issues. They offer limited accuracy, which can require the optimization to go through many more iterations and reduce the efficiency of finding a true optimum [25]. Numerical stability issues also exist when finite differencing NPSS to compute derivatives, which Geiselhart et al. [26] identified as a major motivation for applying gradient-free methods in their low-boom design optimization of a supersonic business jet. Similarly, Allison et al. [27-29] also noted problems with stability and convergence in their extensive work on integrating NPSS into the conceptual design for military aircraft. These issues motivate us to move away from finite-difference derivative approximations and develop a new propulsion analysis tool based on more accurate analytic derivatives.

Future work will first use this propulsion analysis tool to apply gradient-based optimization to propulsion-only models to minimize specific fuel consumption or emissions, and it will later use coupled propulsion-airframe models to minimize mission fuel burn or total energy consumption. Before a new propulsion analysis tool can be built, we must develop a core thermodynamics model capable of providing analytic derivatives. The thermodynamics model computes all properties of the working fluid (enthalpy, entropy, temperature, pressure, density, etc.) given any two thermodynamic states and is a fundamental building block in a full propulsion analysis framework. This model must provide analytic derivatives of computed gas properties with respect to the prescribed properties (e.g., $\partial \gamma / \partial T, \partial S / \partial P$ ), which are then used to compute the derivative for the full propulsion model.

The core thermodynamics model is implemented in the OpenMDAO framework, which uses a Newton solver to converge the nonlinear chemical-equilibrium equations and facilitates the computation of both the derivatives for this Newton solver and the derivatives required for gradient-based optimization via analytic methods (both direct and adjoint) [11,30-32].

The rest of the paper is organized as follows: Sec. II reviews the available thermodynamics methods and justifies the use of the chemical-equilibrium-based method. Section III summarizes the Gibbs energy equilibrium equations and the numerical solver used to converge the chemical-equilibrium equations. The details of the coupled-derivative implementation are given in Sec. IV, and Sec. V verifies the results of the analysis and computations of the derivatives. Finally, Sec. VI presents the optimization results for maximum combustion temperature; the results demonstrate the speed and improvements in numerical stability achieved by using analytic derivatives.

\section{Existing Thermodynamics Tools}

A number of different methods exists for computing the thermodynamic properties of air and air-fuel mixtures, and many implementations of these methods are available. NPSS provides a set of thermodynamics libraries (CEA, JANAF, ALLFUEL, GasTbl), which the user can select at runtime [24]. ALLFUEL and GasTbl are computationally efficient but are based on interpolated tabular data and are only accurate for jet-A fuels or other fuels that are chemically very similar to jet-A fuel. Tran and Snyder [33] demonstrated that tabular thermodynamics data offer limited accuracy when considering fuels that are chemically dissimilar from those used to generate the data. They recommended either generating new tabular data when switching fuels or integrating a chemical-equilibrium analysis directly into the propulsion code. For unconventional cycle configurations that use multiple fuels (for example, a combination of jet-A fuel and liquid natural gas [34]), tabular thermodynamics data are not suitable.

A more advanced technique for building a kriging surrogate model of the equilibrium gas composition was proposed by Walter and Owen [35] and offers a more flexible option for an interpolationbased method. However, this technique does not provide a means of computing the thermodynamic properties of the composite gas. The CEA and JANAF libraries use a chemical-equilibrium-based method, but the JANAF library only considers a fixed set of species. The CEA [36] library is by far the most general of the options in NPSS, accepting an arbitrary number of chemical species that enables it to consider a wide range of fuels.

There is also a number of chemical-equilibrium libraries developed for fields outside of cycle analysis. MINEQL+ [37] specializes in equilibrium reactions in aqueous solutions. ChemSage [38] supports reactions that include metallic elements and metal oxides. JANAF, CEA, MINEQL+, and ChemSage all solve for chemical equilibrium directly by minimizing the Gibbs energy. These codes differ primarily in the details of how they converge the nonlinear chemical-equilibrium equations and offer specialized features for their specific applications. Another alternative is the nonstoichiometric equilibrium method [39], which is used by the open-source library Cantera [40]. Chin et al. [41] used the Cantera implementation for an application involving cycle analysis.

For each propulsion analysis, the thermodynamics module is called hundreds of times, so computational efficiency is important. However, flexibility to consider a wide range of fuels is also important to allow alternative fuels to be investigated, such as hydrogen [42], natural gas $[34,43]$, and biofuels. The low flexibility of interpolative methods makes them less desirable, despite their simplicity. Chemicalequilibrium methods provide the needed flexibility. The Cantera library was ruled out for our purposes due to our prior experience using it in this application, where it led to problems regarding performance. We considered the option of differentiating one of the other chemicalequilibrium libraries, but none of them implements a continuous solution algorithm that is differentiable. The CEA and JANAF libraries 
use a method for handling trace species (those with negligible concentrations) where a heuristic controls their addition and removal from the equilibrium calculations, causing small discontinuities in the solution. These discontinuities are problematic from an analysis point of view [44] and create points where derivatives are not defined. Therefore, we developed a new algorithm for solving the chemicalequilibrium analysis that is differentiable, and thus suitable for gradient-based optimization.

\section{Chemical-Equilibrium Equations}

\section{A. Thermodynamic Properties Prediction}

To find the thermodynamic state of a gas, CEA uses the multistep process illustrated in Fig. 1 as an extended design structure matrix (XDSM) diagram [45]. First, it solves a system of nonlinear equations to minimize the Gibbs energy, which gives the equilibrium composition for the gas at the prescribed state. Second, it solves an additional set of equations to compute the thermodynamic state of the gas by using the converged equilibrium composition. These equations were documented in detail by Gordon and McBride [36] in their seminal paper on CEA. However, the form of the equations presented in their work combined the residuals from the Gibbs energy minimization with a customized procedure for solving for the Newton update. This combination was motivated by the limitations imposed at the time by computer hardware. With modern computers and more powerful linear algebra libraries, it is much better to keep the residuals separate from the numerics to simplify the task of differentiating the analysis. The purely physical form of the equations is presented here. We refer the reader to the original CEA publication for details on the second step, where the thermodynamic properties were computed from the converged equilibrium solution; it remains unchanged from the original formulation [36].

\section{B. Gibbs Energy Minimization}

The full thermodynamic state of a real gas can be defined by any two of the five physical state variables: temperature $T$, pressure $P$, density $\rho$, entropy $S$, and enthalpy $h$. Three specific combinations are useful for propulsion analysis: temperature and pressure $T P$, enthalpy and pressure $h P$, and entropy and pressure $S P$. The $T P$ formulation is the most fundamental. The $h P$ and $S P$ formulations augment the $T P$ formulation equations with a new state variable $T$ and the associated residual to drive the solution to the prescribed value of $h$ or $S$.

\section{Temperature-Pressure Formulation}

The Gibbs energy $g$ is defined as

$$
g=\sum_{j}^{N_{s}}\left(\mu_{j} n_{j}\right)
$$

where $N_{s}$ is the number of chemical species, and $n_{j}$ and $\mu_{j}$ are the concentration (kilogram-mole per kilogram-mixture) and the chemical potential of the $j$ th species, respectively. The chemical potential is a function of temperature, pressure, and concentration; and it is given by

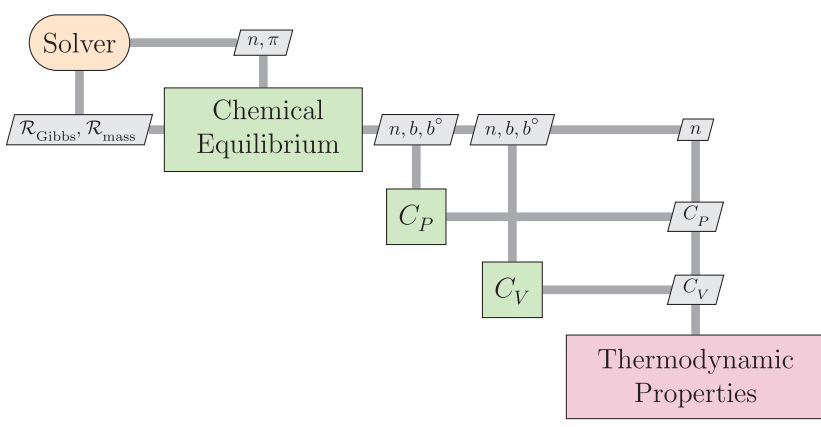

Fig. 1 XDSM diagram of the chemical equilibrium analysis sequence.

$$
\frac{\mu_{j}}{R T}=\frac{H_{j}^{\circ}(T)}{R T}-\frac{S_{j}^{\circ}(T)}{R T}+\ln \left(\frac{P}{P_{a}}\right)+\ln \left(n_{j}\right)-\ln \left(\sum_{k}^{N_{s}} n_{k}\right)
$$

where $R$ is the universal gas constant, and $H^{\circ}$ and $S_{j}^{\circ}$ are given by

$$
\frac{H_{j}^{\circ}(T)}{R T}=\frac{-c_{0}}{T^{2}}+\frac{c_{1} \ln (T)}{T}+c_{2}+\frac{c_{3}}{2} T+\frac{c_{4}}{3} T^{2}+\frac{c_{5}}{4} T^{3}+\frac{c_{6}}{5} T^{4}+\frac{c_{7}}{T}
$$

$$
\frac{S_{j}^{\circ}(T)}{R}=\frac{-c_{0}}{2 T^{2}}-\frac{c_{1}}{T}+c_{2} \ln (T)+c_{3} T+\frac{c_{4}}{2} T^{2}+\frac{c_{5}}{3} T^{3}+\frac{c_{6}}{4} T^{4}+c_{8}
$$

where $c_{0}, \ldots, c_{8}$ are constants for each species, taken from the NIST-JANAF Thermochemical Tables [46].

The equilibrium composition is defined by the values of the concentration variables $n$ that minimize the Gibbs energy subject to conservation-of-mass constraints. The mass is tracked on an elemental basis and is given by

$$
\mathcal{R}_{\text {mass } i}=\sum_{j=1}^{N_{s}}\left(a_{i j} n_{j}\right)-b_{i}^{\circ}=0
$$

where $N_{s}$ is the number of species in the mixture, $b_{i}^{\circ}$ is the amount of each element in the initial composition, and $a_{i j}$ is the stoichiometric constant for element $i$ of species $j$. The mass balance residuals $\mathcal{R}_{\text {mass } i}$ yield $N_{e}$ constraints: one per element present in the mixture. To solve the mass-constrained minimization of the Gibbs energy, we form a Lagrangian

$$
G=\sum_{j=1}^{N_{s}}\left(\mu_{j} n_{j}\right)+\sum_{i=1}^{N_{e}} \lambda_{i}\left(\sum_{j=1}^{N_{s}}\left(a_{i j} n_{j}\right)-b_{i}^{\circ}\right)
$$

where $\lambda_{i}$ is the Lagrange multiplier for the $i$ th element. We differentiate with respect to the $n$ and $\lambda$ variables to build a system of nonlinear equations for which the solution minimizes the Lagrangian:

$$
\delta G=\sum_{j=1}^{N_{s}}\left(\mu_{j}+\sum_{i=1}^{N_{e}}\left(\lambda_{i} a_{i j}\right)\right) \delta n_{j}+\sum_{i=1}^{N_{e}}\left(\sum_{j=1}^{N_{s}}\left(a_{i j} n_{j}\right)-b_{i}^{\circ}\right) \delta \lambda_{i}=0
$$

Because $\delta G$ is linear in $\delta n_{j}$ and $\delta \lambda_{i}$, we can split Eq. (7) into two sets of equations. We get $N_{s}$ equations:

$$
\mathcal{R}_{\text {Gibbs }}=\mu_{j}+\sum_{i=1}^{N_{e}}\left(\lambda_{i} a_{i j}\right)=0
$$

as residuals representing the Gibbs energy. For convenience, because of the factors of $1 / R T$ in Eqs. (2) and (4), we define an alternative Lagrange multiplier as follows:

$$
\pi_{i}=-\frac{\lambda_{i}}{R T}
$$

This yields an alternate form of Eq. ( $\underline{8})$ :

$$
\mathcal{R}_{\text {Gibbs }}=\frac{\mu_{j}}{R T}-\sum_{i=1}^{N_{e}}\left(\pi_{i} a_{i j}\right)=0
$$

Equations (5) and (10) yield a system with $N_{s}+N_{e}$ unknowns and $N_{s}+N_{e}$ residual equations, which can then be solved numerically. 


\section{Enthalpy-Pressure Formulation}

The $h P$ formulation retains the state variables $(n$ and $\pi)$ from the $T P$ solver and the associated residuals from Eqs. (5) and (10). In addition, it adds a new state variable $T$ and a new residual to drive the computed enthalpy to match the specified enthalpy value $h_{0}$ :

$$
\mathcal{R}_{h}=h_{0}-\sum_{j=1}^{N_{s}}\left(n_{j} H_{j}^{\circ}(T)\right)=0
$$

\section{Entropy-Pressure Formulation}

Like the $h P$ problem, the $S P$ formulation uses the same setup as the $T P$ problem with one additional state variable and residual. In this case, the new state variable is the prescribed entropy $S_{0}$. The new residual drives the computed entropy to match the prescribed entropy:

$$
\mathcal{R}_{S}=S_{0}-R \sum_{j=1}^{N_{s}}\left\{n_{j}\left(\frac{S_{j}^{\circ}(T)}{R}-\ln \left(\frac{P}{P_{a}}\right)-\ln \left(n_{j}\right)+\ln \left(\sum_{k}^{N_{s}} n_{k}\right)\right)\right\}=0
$$

where the pressure term is nondimensionalized by standard atmospheric pressure ( $\left.P_{a}=1.01325 \mathrm{bar}\right)$. The reference condition is necessary because entropy is defined as a variation from a reference condition.

\section{CEA Modified Newton's Method for Chemical Equilibrium}

1. Newton Convergence Scheme

Gordon and McBride [36] applied Newton's method to converge the chemical-equilibrium system. When applied to Gibbs energy minimization, Newton's method consists of successive solutions of the linear system

$$
\frac{\partial \mathcal{R}}{\partial U} \Delta U=-\mathcal{R}(U)
$$

where $U=[n, \pi]$ for a $T P$ problem, and $U=[n, \pi, T]$ for $h P$ and $S P$ problems. $\Delta U$ is iteratively computed and applied until the residuals [Eqs. (5), (10-12)] converge to zero within a chosen tolerance.

Note that Eq. (10) involves computing $\mu_{j}$, which through Eq. (2) requires taking the natural logarithm of $n$. In addition, Eq. (4) involves taking the natural logarithm of $T$. Thus, neither $n$ nor $T$ can be negative during the iterations. The natural logarithms also cause numerical difficulties because the derivative with respect to $n$ tends to infinity as $n$ tends to zero. In addition, this means that the Newton system becomes ill conditioned as $n$ tends toward zero. In CEA, this problem is partially dealt with via a logarithmic transformation, where $\Delta U$ is split into two parts: one for $[n, T]$ and another for $\pi$. The $[n, T]$ components of the updates are treated as $\Delta \ln (n) / n$ and $\Delta \ln (T) / T$. The Newton update equation is modified to account for this as follows:

$$
\begin{aligned}
& n_{k+1}=n_{k} \exp \left(\frac{\Delta \ln (n)}{n}\right) \\
& T_{k+1}=T_{k} \exp \left(\frac{\Delta \ln (T)}{T}\right)
\end{aligned}
$$

By using the exponential update form, negative values from the Newton solution are converted into multiplicative updates that are always positive; so, assuming a positive initial guess, the values of $n$ and $T$ never become negative. The $\pi$ update variables are treated normally, with the Newton update

$$
\pi_{k+1}=\pi_{k}+\Delta \pi
$$

Although the CEA method deals with the need to keep both $n$ and $T$ positive, it does not solve the problem whereby trace species create poorly conditioned Jacobians. For this problem, species with $n$ lower than a set value $\left(10^{-5}\right.$ by default) are discarded from the solution. Removal of species, even trace species, introduces a nondifferentiable discontinuity that needs to be avoided. Leal et al. [44] proposed a method that retained all species and modified the computed Newton step for trace species to keep them positive. Because this method retained all species, it was continuous, and thus differentiable. We adopt this method for this work and use a steplimiting method similar to that used by the built-in solvers in OpenMDAO.

\section{Computing $\partial \mathcal{R} / \partial U$}

We compute $\partial \mathcal{R} / \partial U$ analytically, where the nonzero elements for the $T P$ residual partial derivatives are given by

$$
\begin{gathered}
\frac{\partial \mathcal{R}_{\text {Gibbs } j}}{\partial n_{k}}= \begin{cases}\frac{-1}{\sum_{l}^{N_{s}} n_{l}} & \text { if } j \neq k \\
\frac{1}{n_{k}}-\frac{-1}{\sum_{l}^{N_{s}} n_{l}} & \text { if } j=k\end{cases} \\
\frac{\partial \mathcal{R}_{\text {Gibbs } j}}{\partial \pi_{i}}=-a_{i j} \\
\frac{\partial \mathcal{R}_{\text {mass } i}}{\partial n_{j}}=a_{i j}
\end{gathered}
$$

When solving an $h P$ or $S P$ problem, additional nonzero partial derivatives of Eq. ( $\underline{8})$ with respect to $T$ are given by

$$
\frac{\partial \mathcal{R}_{\mathrm{Gibbs} j}}{\partial T}=\frac{\partial H_{j}^{\circ}}{\partial T}-\frac{\partial S_{j}^{\circ}}{\partial T}
$$

For an $h P$ problem, the residual [Eq. (11)] contributes the following nonzero partial derivatives:

$$
\begin{gathered}
\frac{\partial \mathcal{R}_{h}}{\partial n_{j}}=-R T H_{j}^{\circ} \\
\frac{\partial \mathcal{R}_{h}}{\partial T}=-R T \sum_{j=1}^{N_{s}} n_{j}\left(\frac{\partial H_{j}^{\circ}}{\partial T}+H_{j}^{\circ}\right)
\end{gathered}
$$

Similarly, for an $S P$ problem, the residual [Eq. (12)] contributes the following nonzero partial derivatives:

$$
\begin{gathered}
\frac{\partial \mathcal{R}_{S}}{\partial n_{j}}=-R\left[\frac{S_{j}^{\circ}(T)}{R}-\ln \left(\frac{P}{P_{a}}\right)-\ln \left(n_{j}\right)+\ln \left(\sum_{k}^{N_{s}} n_{k}\right)-1\right] \\
\frac{\partial \mathcal{R}_{S}}{\partial T}=-R \sum_{j=1}^{N_{s}}\left(n_{j} \frac{\partial H_{j}^{\circ}}{\partial T}\right)
\end{gathered}
$$

All these nonzero terms can be assembled into a matrix, which is then inverted by using a direct method because the size of the matrix is, at most, $\left(N_{s}+N_{e}+1\right)$ by $\left(N_{s}+N_{e}+1\right)$, where the largest term $N_{s}$ is on the order of hundreds of species.

\section{Multidisciplinary Derivatives}

We define multidisciplinary derivatives as the total derivatives of a function (objective or constraint) with respect to the design variables of a problem where the multidisciplinary system is converged. In other contexts, these could also be called total derivatives or coupled derivatives [11]. This work involves a single engineering discipline: thermodynamics. However, the model is built up of multiple components, as seen in Fig. 1, and each component can be thought of as a subdiscipline. In that sense, any derivative of an OpenMDAO 
model is a multidisciplinary derivative. OpenMDAO automatically computes the multidisciplinary derivatives of an arbitrary model, assuming that each component in the model provides its own partial derivatives $[\underline{47,48]}$.

Partial derivatives are computed for a single component (i.e., derivatives of component outputs with respect to its own input variables); they must be provided to the framework for each component. Partial derivatives can be computed analytically or via automatic differentiation $[11,49]$. In this work, analytic expressions are derived by hand for all components. The derivations are straightforward because of the fine-grained breakdown of the analysis into multiple components, where each component consists of a limited amount of code. This style of building up an analysis via a combination of small components is encouraged when using the OpenMDAO framework because it facilitates differentiation.

Computing the required partial derivatives for a given output is done in a manner that is almost identical to the computation of the partial derivatives for the Newton solver. The only difference is that, for the Newton solver, we compute only the partial derivatives of the residual equations with respect to the state variables. To compute the multidisciplinary derivatives, we reuse the derivatives from the Newton solver but now also include partial derivatives with respect to all other inputs of that component. As an example, consider Eq. (12). The two partial derivatives needed for the Newton solver are given by Eqs. (23) and (24). These two derivatives are augmented with the two following additional derivatives:

$$
\begin{gathered}
\frac{\partial \mathcal{R}_{s}}{\partial S_{0}}=1 \\
\frac{\partial \mathcal{R}_{s}}{\partial P}=\frac{R}{P} \sum_{j=1}^{N_{s}} n_{j}
\end{gathered}
$$

Once all the partial derivatives are computed, OpenMDAO automatically assembles them into a linear system that, by using either a coupled direct or a coupled adjoint method [50], is solved to compute the multidisciplinary derivatives. In OpenMDAO, this is achieved via the unified derivative equation [11]:

$$
\frac{\partial \mathcal{R}}{\partial U} \frac{\mathrm{d} u}{\mathrm{~d} r}=\mathcal{I}=\frac{\partial \mathcal{R}}{\partial U} \frac{\mathrm{d} u^{T}}{\mathrm{~d} r}
$$

where $\partial \mathcal{R} / \partial U$ is a Jacobian matrix of partial derivatives, $\mathcal{I}$ is the identity matrix, and $\mathrm{d} u / \mathrm{d} r$ is the matrix of total derivatives for which we want to solve. The left-hand side of Eq. (27) represents the coupled direct method, solved one time per design variable; whereas the right-hand side represents the coupled adjoint method, solved one time per function of interest. The coupled adjoint capability is the most significant, given the ultimate goal of integration with adjoint CFD codes, but once the partial derivatives are given, both direct and adjoint solvers are available from OpenMDAO with no additional work. In Eq. (27), the Jacobian $\partial \mathcal{R} / \partial U$ is similar to that used in the Newton solver from Eq. (13), but it includes the additional partial derivatives with respect to all the inputs, represented by Eqs. (25) and (26) in our case.

For the chemical-equilibrium model developed herein, the structure of $\partial \mathcal{R} / \partial U^{T}$ is shown in Fig. 2 for a $T P$ solver and in Fig. 3 for the $h P$ or $S P$ solver. The diagonal terms are the partial derivatives of the residuals with respect to the associated state variable or an output with respect to itself. The off-diagonal terms are partial derivatives of residuals or outputs with respect to the other variables. In Fig. 2 , the $2 \times 2$ block of partial derivatives, outlined in dark gray, are the same derivatives needed for the Newton solver. These partial derivatives are given by Eqs. (17-19). For the $T P$ solver, temperature is an input to the calculation; but, for the $h P$ and $S P$ solvers, it becomes a state variable. For these slightly more complex solvers, the $\partial \mathcal{R} / \partial U$ matrix includes $h$ or $S$ as inputs and $T$ as an additional state variable. The $3 \times 3$ block, outlined in dark gray in Fig. 3, contains the Newton derivatives for the corresponding solvers. All other shaded boxes outside the Newton blocks are the additional partial derivatives needed to compute the multidisciplinary derivatives. It is convenient

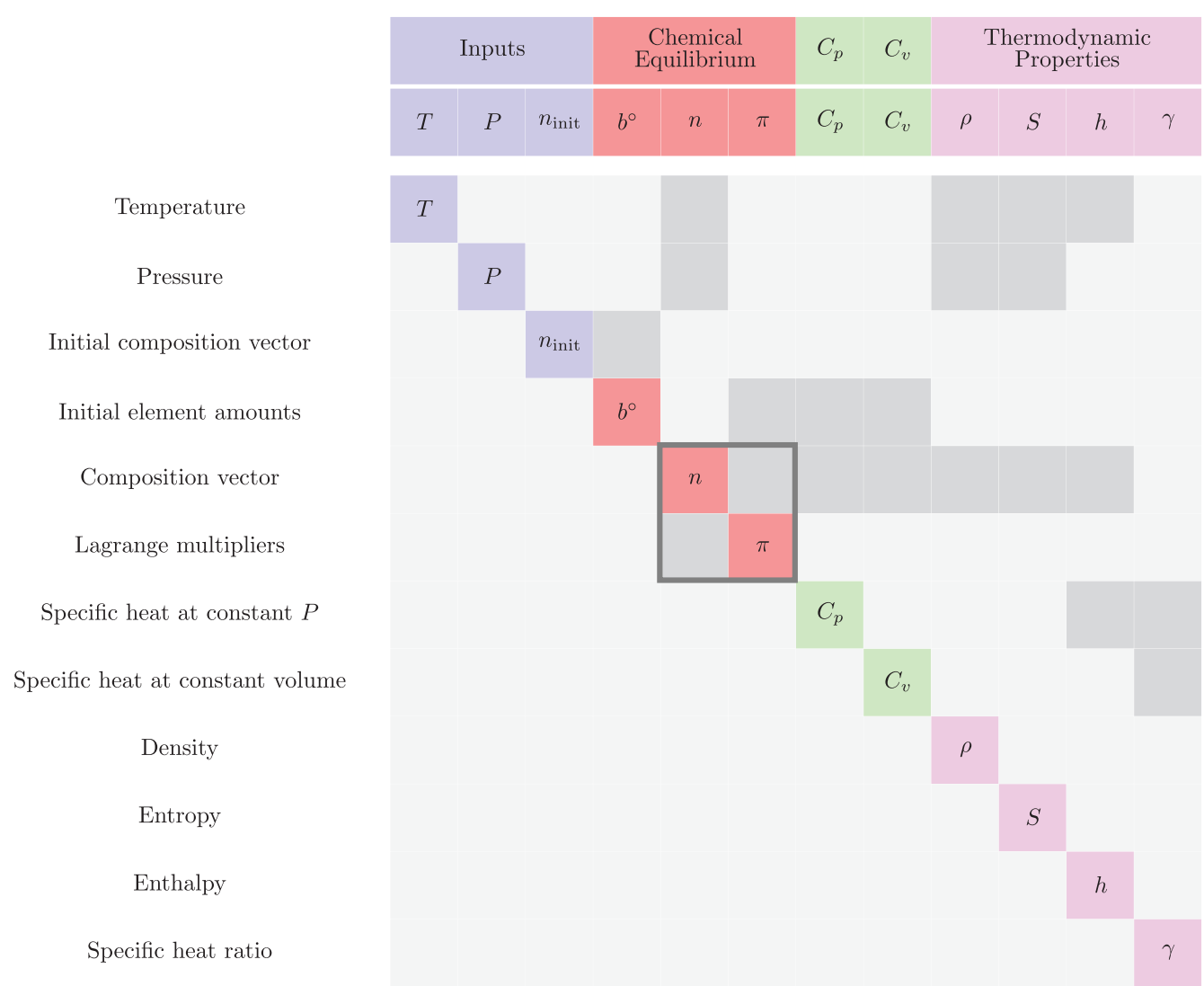

Fig. 2 Structure of partial derivative matrix for adjoint multidisciplinary derivatives of the $T P$ solver. The subset of partial derivatives needed for the Newton solver is highlighted by the gray square outline. 


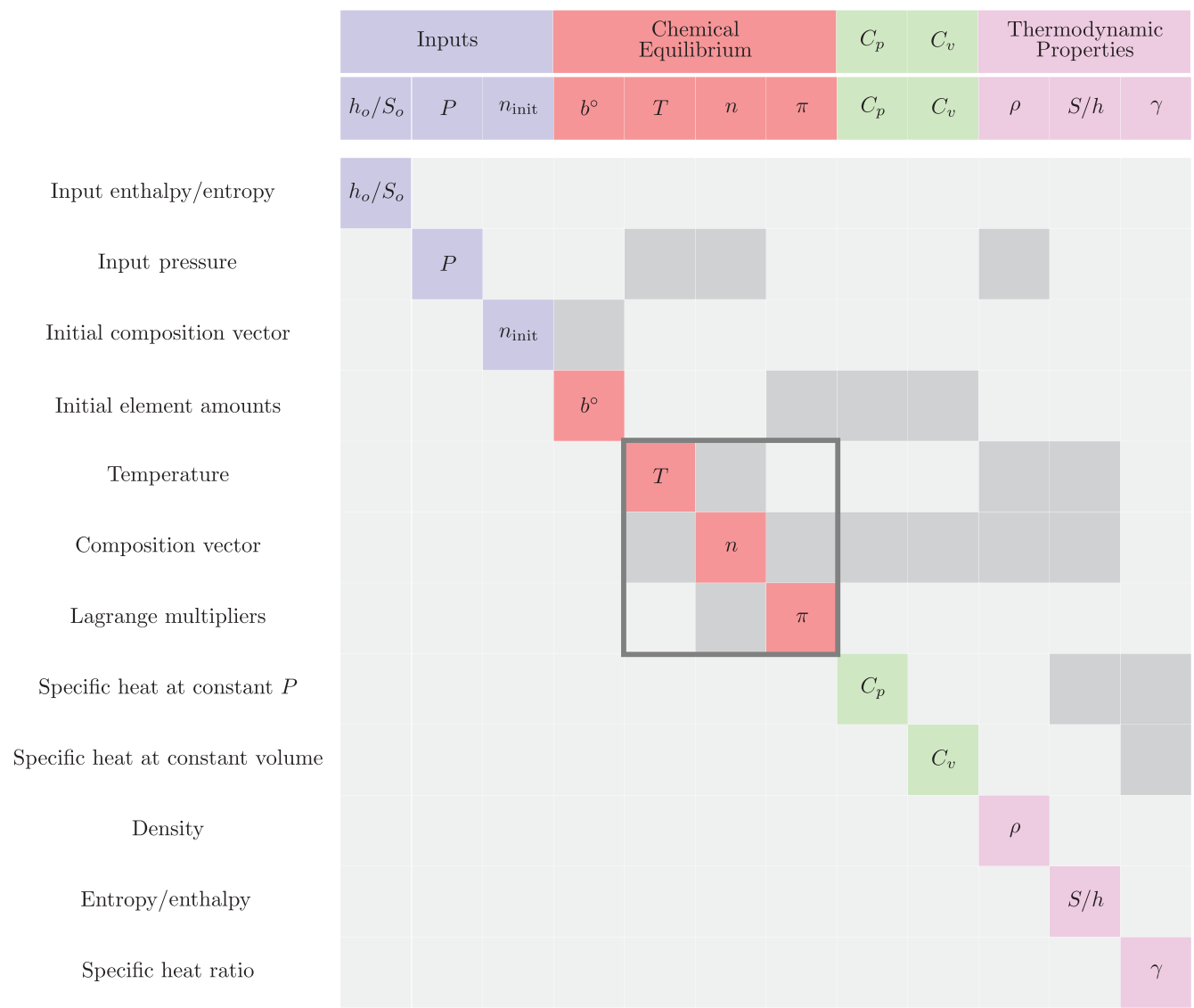

Fig. 3 Structure of partial derivative matrix for adjoint multidisciplinary derivatives of $h P$ and $S P$ solvers. The subset of partial derivatives needed for the Newton solver is highlighted by the gray square outline.

that the partial derivatives necessary for a Newton solver can be reused with the unified derivative equations, but Figs. 2 and 3 also show that significantly more partial derivatives may be required in the latter case.

Although Eq. (27) is just a linear system, solving it efficiently for a chemical-equilibrium model is not easy. The lower diagonal elements of the matrix prevent the use of Gaussian elimination. A naive implementation could use a direct method to solve the entire linear system monolithically. Although this would work for an isolated chemical-equilibrium analysis, a more complex propulsion model with many such solutions would become prohibitively large for that approach. The modular analysis and unified derivatives (MAUD) architecture for solving the derivative equations suggests a more efficient approach that uses a nested linear solver that follows the hierarchy of the nonlinear model to generate effective preconditioners for use with sparse iterative linear solvers such as the generalized minimum residual (GMRES) method [48].

OpenMDAO implements the MAUD solver architecture by allowing each component to specify its own nonlinear solver and linear solver. Figure 4 illustrates the model hierarchy and shows which linear and nonlinear solvers are used for each component. At the top level, one iteration through each subcomponent is used for the nonlinear solver because there is no intercomponent coupling in this

\begin{tabular}{|c|c|c|c|c|}
\hline \multicolumn{5}{|c|}{$\begin{array}{c}\text { Top Level } \\
\text { NL Solver: One Iteration } \\
\text { LIN Solver: LNGS }\end{array}$} \\
\hline Inputs & $\begin{array}{c}\text { Chemical } \\
\text { Equilibrium } \\
\text { NL: Newton } \\
\text { LIN: GMRES }\end{array}$ & $C_{P}$ & $C_{V}$ & $\begin{array}{c}\text { Thermodynamic } \\
\text { Proproperties } \\
\text { LIN: Direct }\end{array}$ \\
$\begin{array}{c}\text { NL: One Iteration } \\
\text { LIN: LNGS }\end{array}$ & $\begin{array}{c}\text { NL: Direct } \\
\text { LIN: Direct }\end{array}$ & $\begin{array}{c}\text { NL: One Iteration } \\
\text { LIN: LNGS }\end{array}$ \\
\hline
\end{tabular}

Fig. 4 Hierarchy for chemical-equilibrium solver with both nonlinear (NL) and linear (LIN) solvers indicated for each component. model. The sequential nature of the nonlinear solver enables a Gaussian elimination at the top level, implemented by one-block linear Gauss-Seidel (LNGS) iteration for the linear solution. The inputs and thermodynamic properties components are both a collection of explicit equations that can also use a LNGS algorithm with a single iteration for the linear solution. The chemicalequilibrium component uses a Newton solver for the nonlinear solution and a GMRES linear solver. The $C_{P}$ and $C_{V}$ components are composed of linear equations that can be solved by using a direct method for both the nonlinear and the linear solvers, although this component is actually only linear. For chemical equilibrium, $C_{P}$ and $C_{V}$, a natural synergy exists between the nonlinear and linear solvers. For chemical equilibrium, the same linear solver needed to solve for the Newton update can be reused for the derivatives. For $C_{P}$ and $C_{V}$, the exact same solver can be used for both because both are linear systems.

\section{Verification of Analysis and Derivatives}

The thermodynamics module is to be the foundation for a new cycle analysis tool, so it is important that it accurately models the thermodynamic properties of air and air-fuel mixtures across a wide range of temperatures and pressures. Given the goal of using this work for optimization, it is also important that the analytic derivatives be correct. This section presents the verification of the analysis and the corresponding derivatives.

\section{A. Approach to Analysis Verification}

The new code was verified against CEA predictions. The verification cases correspond to the temperature and pressure combinations listed in Table 1 . A total of 3600 different conditions were examined with temperatures ranging from 200 to $4800^{\circ} \mathrm{R}$ and pressures from 1 to $1500 \mathrm{psi}$. This regular grid was run at four equivalence ratios $\phi$ of $0,0.015,0.3$, and 0.44 to provide a wide range of combustion conditions. The equivalence ratio (the ratio of the 
Table 1 Temperature and

pressure conditions used for verification cases

\begin{tabular}{lccc}
\hline \hline & Low & High & Step \\
\hline Temperature, $^{\circ} \mathrm{R}$ & 200 & 4800 & 200 \\
Pressure, psi & 1 & 1500 & 10 \\
\hline \hline
\end{tabular}

actual fuel-to-air ratio to the stoichiometric value) is a convenient way to express the amount of potential combustion in a manner that is independent of the specific fuel being used. This verification grid includes low temperatures that are not physically meaningful and that actually extend below the valid range of the thermodynamics data provided as input. Such low temperatures are run, not to test the physical predictive power of the code under invalid conditions but rather to compare it numerically with CEA under extreme conditions. Because the new code is to be used for optimization, it must be numerically stable even under nonphysical conditions because optimizations often iterate through physically invalid areas on their way to the optimum.

To compute the verification data, the new code was set up with air at the temperature and pressure conditions prescribed in Table 1 and then combusted at the given $\phi$ with jet- $\mathrm{A}$, which is a hydrocarbon fuel $\left(\mathrm{C}_{12} \mathrm{H}_{23}\right)$ with a stoichiometric fuel-air ratio of 0.06817 corresponding to $\phi=1$.

The combustion was modeled as a prescribed enthalpy process by computing the overall enthalpy of the air-fuel combination and holding it constant while solving for a chemical-equilibrium composition. Because the total enthalpy calculation was already required for the combustion model in the new code, the corresponding CEA runs were set up with $h$ and $P$ by using the enthalpy output from the corresponding case. For both codes, a reduced set of 19 chemical species was considered in order to reduce the number of trace species present in the converged equilibrium results and to improve computational speed. The following species were included: $\mathrm{N}, \mathrm{NH}_{3}, \mathrm{~N}_{2}, \mathrm{NO}, \mathrm{NO}_{2}, \mathrm{NO}_{3}, \mathrm{CH}_{4}, \mathrm{C}_{2} \mathrm{H}_{4}, \mathrm{CO}, \mathrm{CO}_{2}$, $\mathrm{O}, \mathrm{OH}, \mathrm{O}_{2}, \mathrm{H}, \mathrm{H}_{2}, \mathrm{H}_{2} \mathrm{O}, \mathrm{HO}_{2}, \mathrm{H}_{2} \mathrm{O}_{2}$, and Ar.

Two different types of verification are performed. First, the predicted chemical-equilibrium compositions are compared to ensure that the proper amount of each chemical species is present at each test point. Next, the actual thermodynamic properties $(P, T, \rho, h$, $\left.S, C_{P}, C_{V}, \gamma\right)$ are compared. To verify the composition, the average discrepancy between the codes is $5.2 \times 10^{-6} \mathrm{~mol}$ and the maximum error is $1.1 \times 10^{-4} \mathrm{~mol}$. The average discrepancy in the prediction of the thermodynamic properties is $0.03 \%$, and the maximum error is $0.52 \%$. These results demonstrate a strong agreement over a wide range of temperatures and pressures.

One unavoidable source of discrepancy between the new code and CEA comes from implementation details related to handling trace species, as discussed in Sec. III.C.1. Figure 5 quantifies this effect more clearly, showing the number of species retained in the final

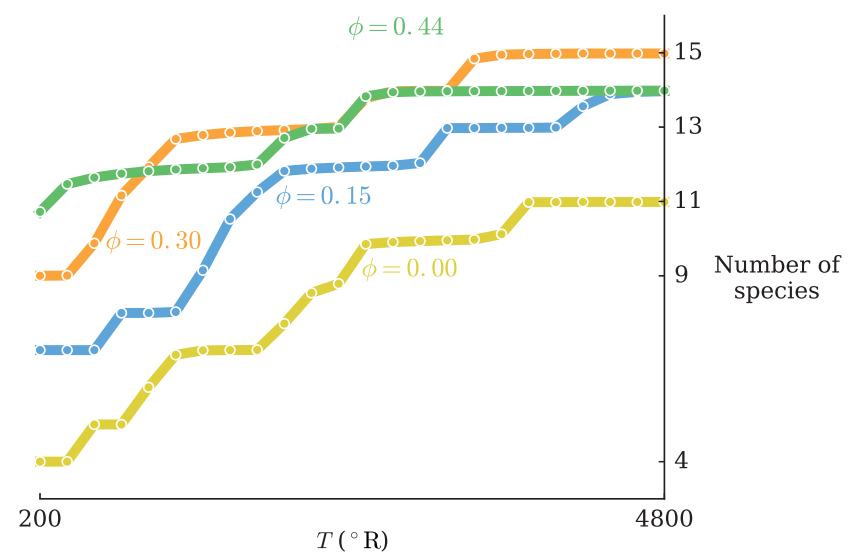

Fig. 5 Variation of total number of active chemical species in CEA solutions with respect to temperature. solution from CEA for different values of $\phi$ over a range of temperatures. The number of active species varies most for $\phi=0$ (from 4 to 11) because, at lower temperatures, the composition of air stays relatively close to atmospheric, but $\mathrm{NO}_{x}$ starts to form and dissociation starts to occur at the higher temperatures. Note that the data in Fig. 5 are pressure averaged over the entire range of pressures from Table $\overline{1}$. A slight negative correlation exists between pressure and the number of active species in the data, but this is negligible compared with the temperature effect. If a larger set of species were considered, the pressure effect could be more pronounced.

\section{B. Verification of Chemical Equilibrium}

The first verification serves to confirm that the new code returns the same chemical composition as does CEA over the verification grid from Table 1 . The comparison is done on a per-species basis and is measured with absolute differences. Air, even when combusted with $\phi=0.44$, is composed of over $70 \%$ diatomic nitrogen, which means that any other chemical species (e.g., $\mathrm{CO}_{2}, \mathrm{H}_{2} \mathrm{O}$ ), although important to the thermodynamic properties, makes up less than $30 \%$ of the overall gas. Some species (e.g., $\mathrm{NH}_{3}, \mathrm{NO}_{3}$ ) are present only in trace amounts and have almost no impact on the thermodynamics. The accuracy of the amount of trace species predicted by using a chemical-equilibrium method is highly limited but is still of practical significance to ensure that the two codes predict the same trace species. The low concentration of many species requires an absolute error to measure the discrepancy between codes and, to be meaningful, absolute error requires knowledge of the actual value for $n_{j}$. Figure 6 shows the accuracy of the predicted values for $n$ averaged

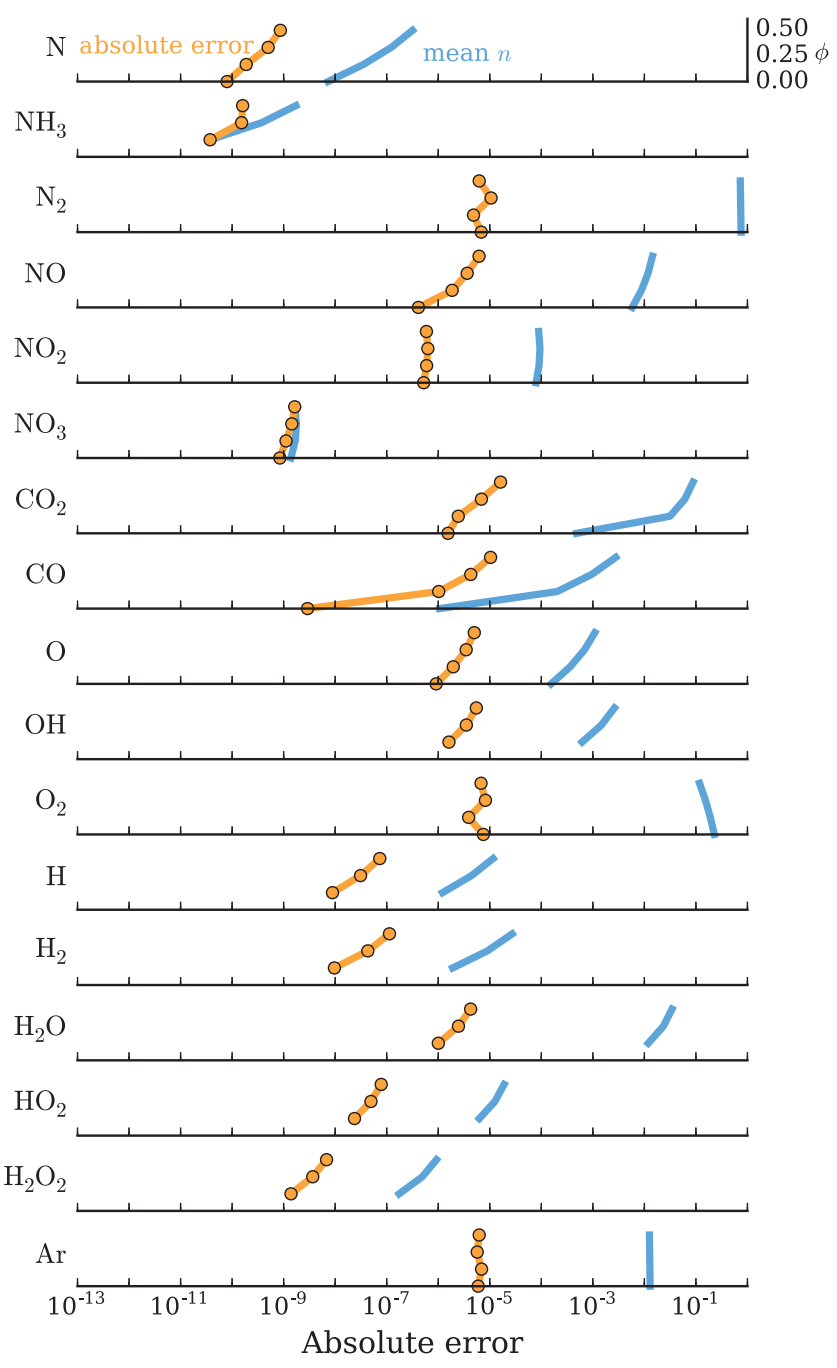

Fig. 6 Mean differences between equilibrium gas compositions predicted by CEA and by the proposed code. 


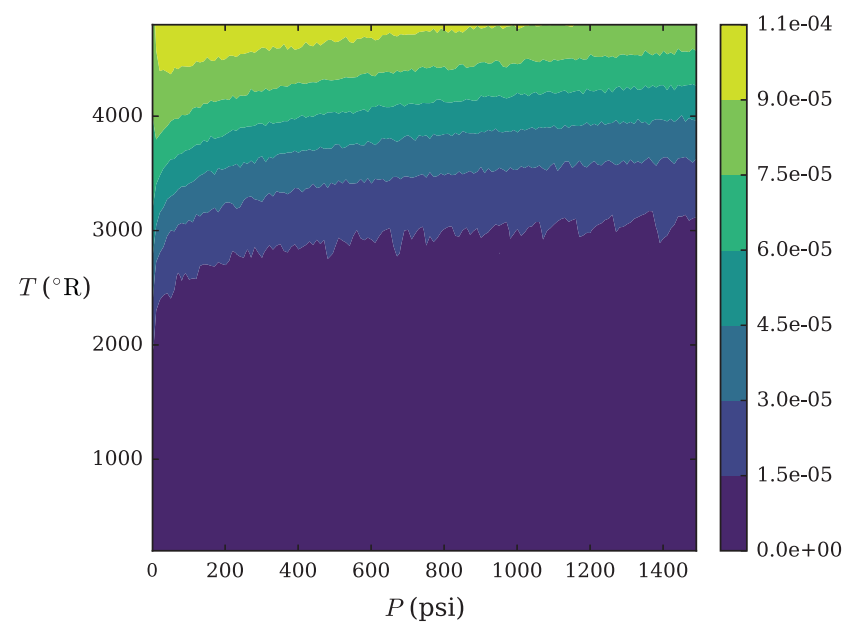

Fig. $7 l^{2}$ norm of difference between concentration vectors $\left\|n_{\mathrm{CEA}}-n\right\|_{2}$.

over the full $T$ and $P$ verification set as a function of equivalence ratio. The mean $n_{j}$ are shown as solid blue lines and can be compared with the absolute errors $\left\|n_{j \mathrm{CEA}}-n_{j}\right\|_{2}$, which are represented as orange lines. Most of the errors are at least two orders of magnitude smaller than the mean concentrations, indicating strong agreement between the two codes. For $\mathrm{NH}_{3}$ and $\mathrm{NO}_{3}$, the discrepancies are the same order of magnitude as the mean value because CEA results include these species at the lower limit of $10^{-10}$. These two species further highlight the subtle difference between the trace-species method for CEA and the new code. At such small concentrations, these species do not have a meaningful impact on the thermodynamic properties. Note that $\mathrm{CH}_{4}$ and $\mathrm{C}_{2} \mathrm{H}_{4}$ are excluded from Fig. $\underline{6}$ because they are always trace species for CEA and their compositions are always below $10^{-13}$ for the new code.

In addition to showing the discrepancy for each species individually, Fig. 7 also shows how the norm of the absolute error $\left\|n_{\mathrm{CEA}}-n\right\|_{2}$ varies with $T$ and $P$ for the case of $\phi=0.44$. There is a clear trend toward increasing discrepancy with higher temperature and a much weaker correlation with increasing pressure. Although the discrepancy grows from $3 \times 10^{-5}$ to $2 \times 10^{-4}$ upon moving from the lower-left corner to the upper-right corner, it is still small. This trend is consistent with Fig. 5, where CEA starts introducing small concentrations of new species as the temperature rises. Because different methods are used to handle these trace species, we expect additional discrepancy in areas where trace species are prevalent. Figures $\underline{6}$ and $\underline{7}$ demonstrate that both codes give results that are in strong agreement in terms of overall composition across the entire verification grid. The overall conclusion is that the proposed code and CEA both compute the same composition within solver tolerance.

From a cycle analysis perspective, the composition vector $n$ is not important in itself; however, because the thermodynamic properties are computed as a function of $n$, it is still important. Therefore, the verification of predicted composition serves as a preliminary verification of the thermodynamics itself. The next section presents the verification of the thermodynamic properties, but these data reinforce those results by ensuring that the calculations are based on the same chemical compositions.

\section{Verification of Thermodynamic Properties}

We use relative measurements to compare the thermodynamic state variables predicted by CEA with those predicted by the new code. Figure 8 shows mean discrepancy, measured across the full verification grid, for entropy $S$, temperature $T$, enthalpy $h$, density $\rho$, pressure $P$, specific heat ratio $\gamma$, and specific heat at constant pressure $C_{P}$. The mean error for all cases, across all properties, is $0.03 \%$, and the maximum error is $0.52 \%$. Note that, because both $h$ and $P$ are set directly for the CEA run, from the output of the corresponding cases with the new code, these properties have the lowest errors in Fig. 8. The other errors are larger but are still less than $0.1 \%$. This demonstrates a strong agreement between CEA and the new code, and it verifies its predictions for cycle analysis applications.

\section{Verification of Multidisciplinary Derivatives}

The derivative accuracy is verified by comparing analytic derivatives (direct method in this case) to finite-difference approximations by using forward-, central-, and backward-difference methods. Figures 9 and 10 show the relative error between the three finite-difference derivative approximations and the analytic reference values. This relative error is plotted for varying finite-difference step sizes. The plots follow the structure of the Jacobian, showing the derivatives of $h, S, \rho, C_{P}$, and $\gamma$ (rows) with respect to $T$ and $P$ (columns). Figure 9 corresponds to standard day conditions ( $288 \mathrm{~K}$, 1 bar), whereas Fig. 10 corresponds to a representative cruise condition $(1500 \mathrm{~K}, 1 \overline{0}$ bar $)$. The analytic and finite-difference

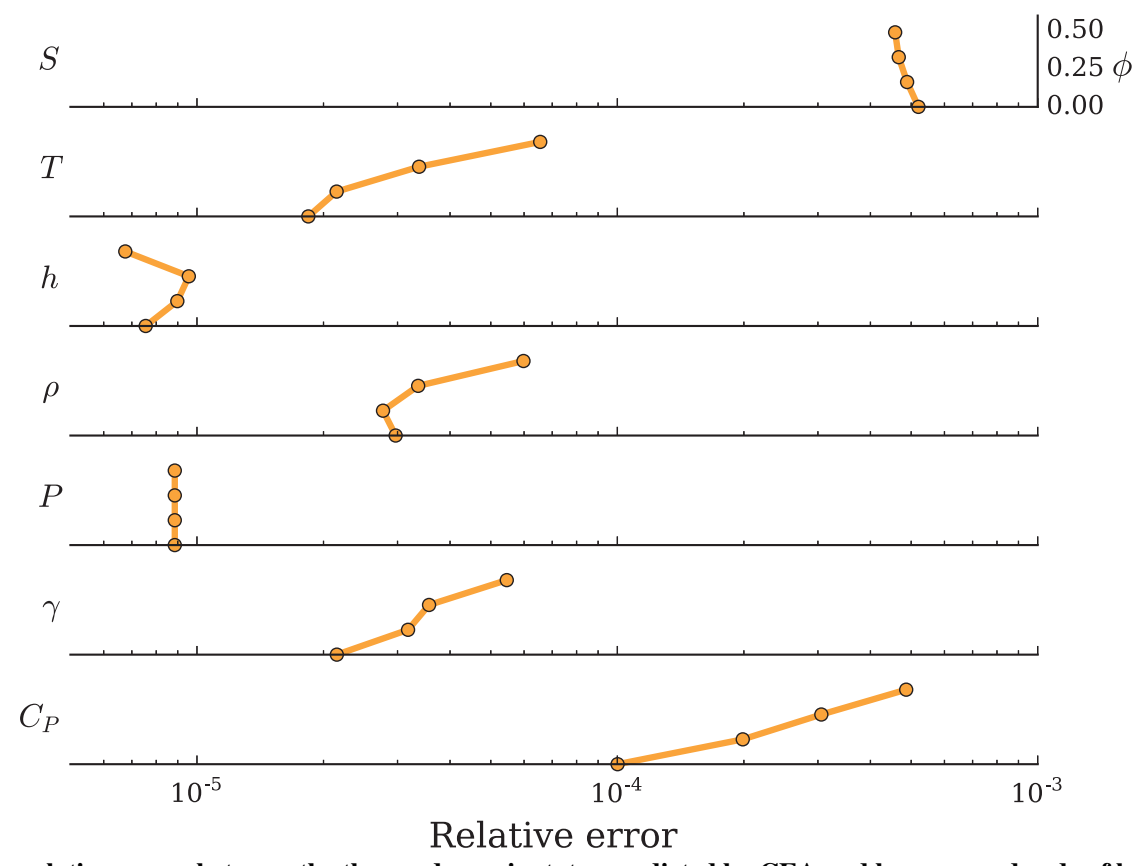

Fig. 8 Mean relative errors between the thermodynamic states predicted by CEA and by proposed code of less than $0.1 \%$. 
$T$
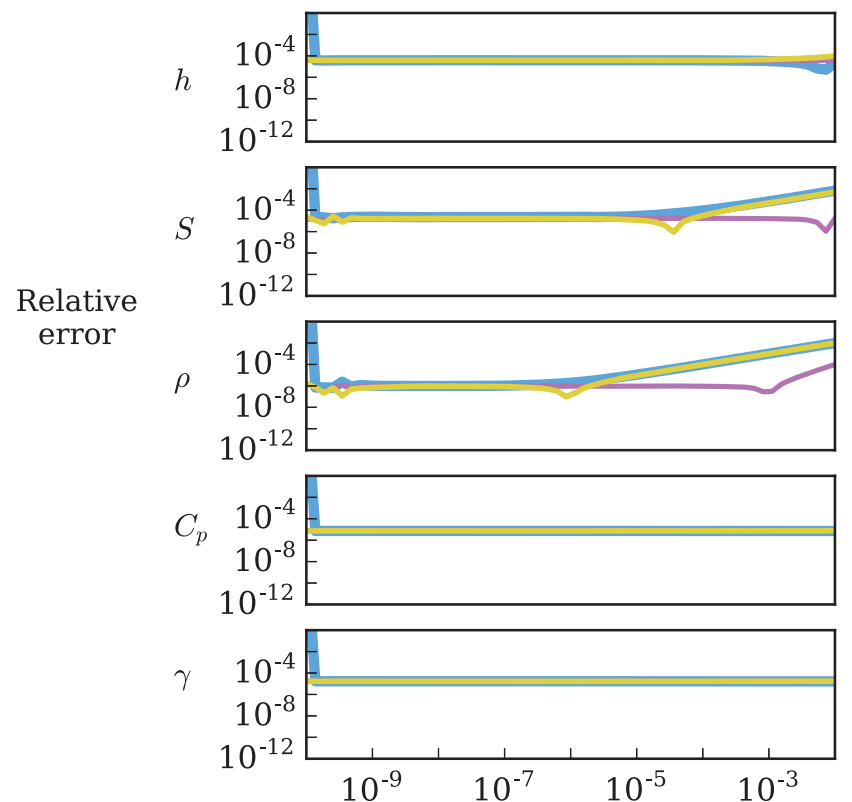

$P$
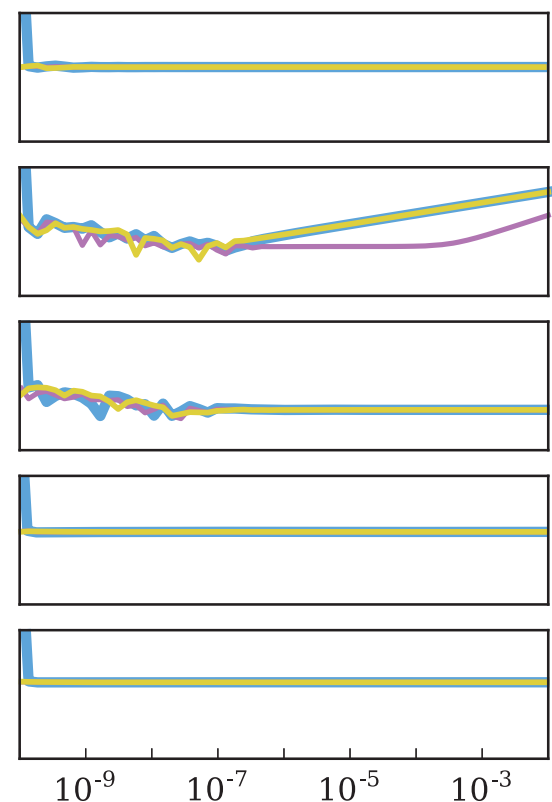

Finite difference step size

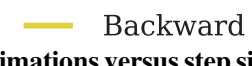

Central

Forward

Fig. 9 Relative error of finite-difference approximations versus step size obtained for standard day conditions and by using computed analytic values as reference.

\section{$T$}
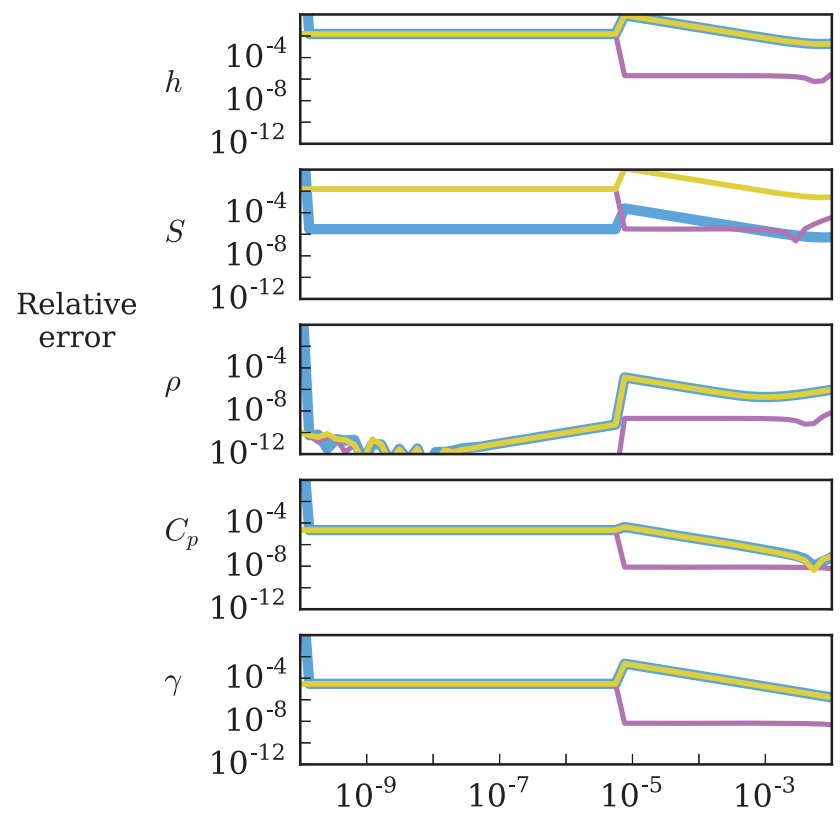

$P$
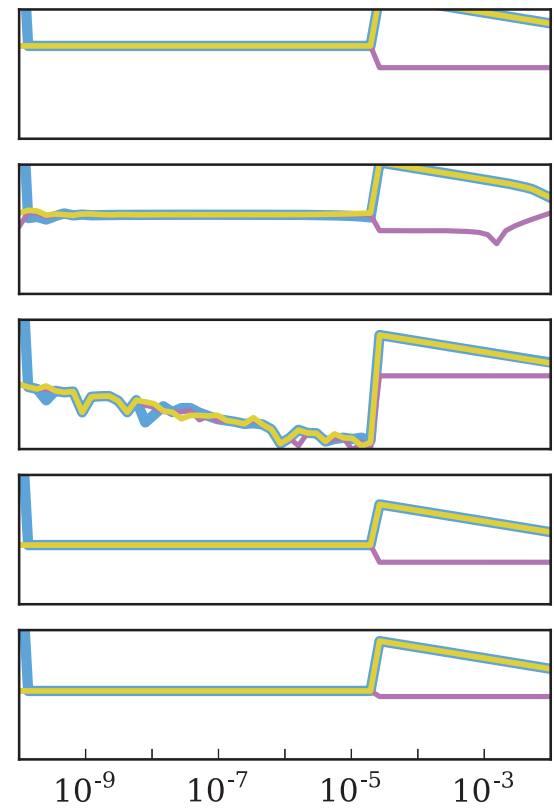

Finite difference step size

\section{Backward}

Central

Forward

Fig. 10 Relative error of finite-difference approximations versus step size for cruise conditions and with computed analytic values as reference.

derivatives all agree to at least $10^{-5}$, although the point of best agreement occurs at different step sizes for different variables.

Whereas the results for standard day conditions in Fig. $\underline{9}$ show good agreement for all step sizes, the results for the cruise condition in Fig. 10 show a dramatic increase in accuracy when the relative step size is less than $10^{-5}$. At cruise conditions, the temperature and pressure are higher, and the converged mixture contains many more active species, which makes the solution more sensitive to input values. Thus, keeping the step size sufficiently small to prevent major changes in the solution is the key to obtaining accurate finitedifference approximations. The central-difference approximation converges faster than the forward or backward schemes and offers better overall accuracy over a range of step sizes. This is expected, because central differencing is second-order accurate, whereas the 


\begin{tabular}{lc}
\multicolumn{1}{c}{ Method } & Time (s) \\
\hline \hline Direct & 0.0046 \\
Adjoint & 0.0085 \\
Forward finite difference & 0.0110 \\
Central finite difference & 0.0213 \\
Backward finite difference & 0.0110
\end{tabular}

Fig. 11 Wall times required to compute the $5 \times 2$ Jacobian by using analytic methods (direct and adjoint) and finite-difference approximations.

other two schemes are first order accurate. However, this accuracy comes at a cost because central differencing requires two function evaluations for each derivative.

Figure 11 shows the computational times for assembling the full $5 \times 2$ Jacobian of the responses with respect to the design variables. All computational times were measured on an Apple MacBook Pro laptop with a $2.6 \mathrm{GHz}$ dual-core Intel Core i5 processor and $16 \mathrm{~GB}$ of memory. The computational cost of the central-difference approximation is twice that of the forward- and backward-difference schemes. Computing the derivatives with the direct method is faster than with the finite-difference approximations because each finitedifference step requires the convergence of the full nonlinear model, as opposed to the solution of a linear system for the direct method.

Of the two analytic methods, the direct method is faster than the adjoint method for this problem. Recall that the direct method corresponds to solving the left-hand side of the unified derivatives [Eq. (27)], whereas the adjoint method solves the right-hand side of the same equation. In this case, the direct method is expected to be faster because it involves two independent variables and five responses. Thus, for the direct method, two linear solutions are needed to compute the full Jacobian. For the adjoint method, five linear solutions are needed. For problems with more design variables than constraints, the adjoint method would become advantageous.

\section{Optimization Results}

\section{A. Equivalence Ratio Optimization}

To demonstrate the use of analytic derivatives in a gradient-based optimization, we ran a series of unconstrained optimizations at a fixed precombustion gas temperature of $518^{\circ} \mathrm{R}$. Each optimization sought to maximize the combustion temperature by varying $\phi$ for a given fixed pressure. We solved 200 optimizations for pressures ranging from 15 to $1500 \mathrm{psi}$. Figure 12 plots the optimal temperatures and the

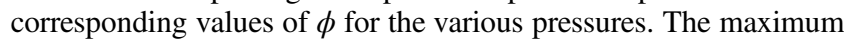
temperatures occurred for $\phi$ between 1.02 and 1.07, which seemed counterintuitive. Under the assumption of perfect combustion, the maximum temperature would occur at the stoichiometric value, $\phi=1$, where every molecule of diatomic oxygen would be
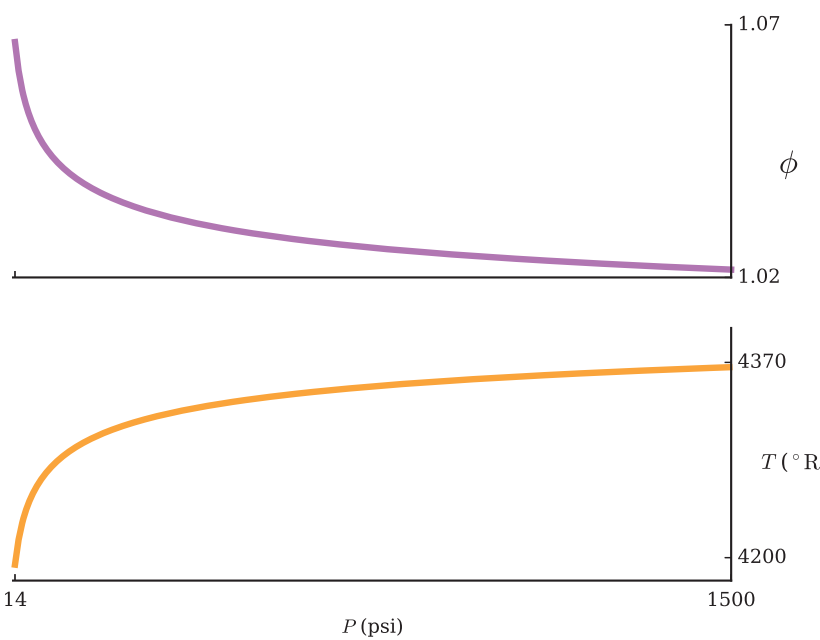

Fig. 12 Air-fuel equivalence ratio $\phi$ that maximizes the combustion temperature $T$ as a function of pressure $P$ ranging from 15 to $1500 \mathrm{psi}$. converted to water. However, equilibrium calculations took into account disassociation effects, which simultaneously lowered the maximum achievable temperature and caused that temperature to occur at a richer $\phi$ [51]. The effect of dissociation became less severe at higher pressures, which tended to favor the creation of slightly larger molecules, and the reaction more closely approximated ideal combustion. This is shown in Fig. 12 by both the increasing maximum temperature and the decreasing optimal $\phi$ as pressure increased. These optimization problems were solved by using gradients computed with both the finite-difference method and the adjoint method. The results were identical in terms of both overall execution time and final objective value because, for this problem with a single design variable, the adjoint method did not significantly improve the speed or accuracy.

\section{B. Equivalence Ratio and Pressure Optimization}

These results establish that combustion becomes more efficient as pressure increases, even when accounting for equilibrium chemistry effects. Therefore, we expect that including pressure as a design variable yields the same result without requiring the parameter study for pressure. To verify this, a second series of optimizations is solved with both $\phi$ and $P$ as design variables, again seeking to maximize combustion temperature. For this set of optimizations, the tolerance of the chemical equilibrium numerical solver is varied from $10^{-8}$ to $10^{-12}$ to test how sensitive the optimization is to the accuracy of the solution. Again, we use two methods to compute derivatives: the adjoint method and the forward finite-difference approximation. Both derivative methods converge to $\phi=1.021$ and $P=1500 \mathrm{psi}$, which is consistent with the previous optimization results shown in Fig. 12. However, unlike the single-design-variable optimization, the adjoint method clearly performs better than the finite-difference method. The finite-difference approximation is at least twice as expensive as the adjoint method, as shown in Fig. 13. One of the main challenges with using finite-difference derivatives is the need to have tight tolerances for the solvers, such as the one used to converge chemical equilibrium. The data in Fig. 13 quantify this effect by comparing the performance for different tolerances of chemical equilibrium solvers. The optimizations that use adjoint-computed gradients require a nearly constant computation time. However, the optimizations that use finitedifference derivatives require between 5.5 and $110 \mathrm{~s}$, depending on solver tolerance and finite-difference step size. This wide range in computational time results from inaccurate derivatives, which force the optimizer to iterate more to converge to the required tolerance. The worst performance occurs when using a step size of $10^{-6}$ with tolerances between $10^{-11}$ and $10^{-10}$, but the times are reduced for step sizes between $10^{-10}$ and $10^{-9}$. This erratic behavior is particularly troubling and shows that finite-difference approximations are not reliable. For both step sizes, with a solver tolerance of $10^{-8}$, the computational times start to rise. Beyond that point, numerical noise

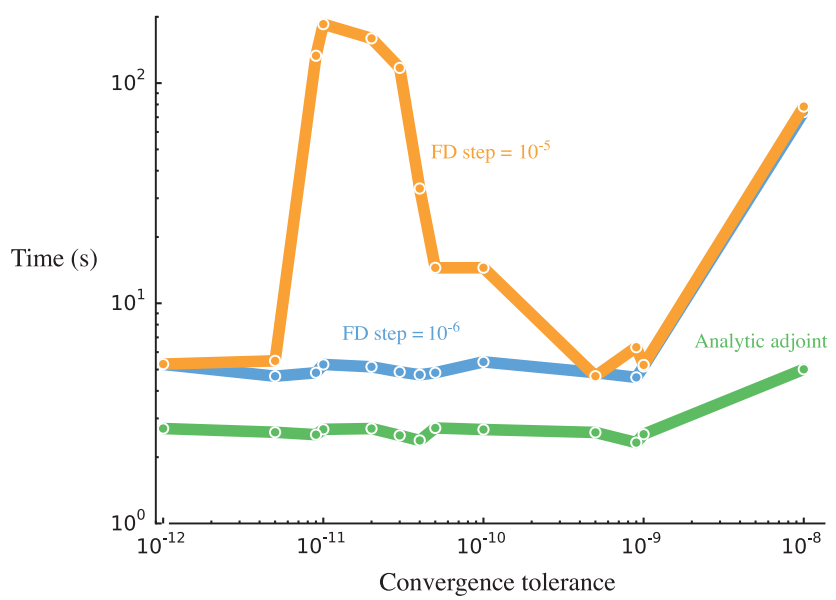

Fig. 13 Optimization wall time versus tolerance of chemical equilibrium solver obtained by using analytic and finite-difference (FD) methods to compute derivatives. 
prevents the optimizer from converging when using finite-difference derivatives. This result highlights the value of the analytic approach. Even for just two design variables, the adjoint derivatives enable both faster and more stable optimization.

\section{Conclusions}

The need to incorporate propulsion cycle modeling into the multidisciplinary design optimization (MDO) of aircraft motivates the development of a new propulsion cycle analysis model that can efficiently compute derivatives. As a first step toward this new capability, a new chemical equilibrium thermodynamics method is developed that predicts the gas properties of air and fuel-air mixtures for a wide range of fuels in a computationally efficient manner. The major contribution of the new method is the addition of analytic derivative computation (both direct and adjoint). To compute the coupled derivatives, it is necessary to reformulate the mechanism for handling trace species in the chemical equilibrium solver to create a continuous analysis response.

The new method was implemented using the OpenMDAO framework and its automatic coupled-derivatives capability to simplify the development. This implementation was verified by comparing its results against those obtained with the CEA code over a wide range of temperatures, pressures, and equivalence ratios. The verification results match closely, with a maximum discrepancy between the proposed implementation and CEA of less than $0.1 \%$. In addition to validating the analysis, the accuracy of the analytic derivatives was verified by comparing them to finite-difference approximations. The analytic derivatives agreed with the finite-difference approximations to within a relative error of $O\left(10^{-5}\right)$.

The value of the analytic derivatives approach is further demonstrated via two optimizations for which the goal is to maximize the combustion temperature with respect to the pressure and equivalence ratio. These optimizations compare the performance of the adjoint derivatives with that of the finite-difference derivatives and show that, even for an optimization with only two design variables, adjoint derivatives significantly reduce the computational cost and increase the numerical stability. The improvements in speed and accuracy clearly demonstrate the value of the adjoint derivatives for optimization applications and suggest that similar improvements in performance for larger propulsion cycle analyses are possible.

Future work will consist in building a new propulsion analysis method based on this thermodynamics method. Models for inlets, compressors, combustors, turbines, and nozzles will be developed with adjoint derivatives, and then combined into full propulsion models.

\section{Acknowledgments}

We would like to thank the NASA Transformational Tools and Technologies Project, under the Aeronautics Research Mission Directorate, for funding this work. We would also like to thank Kenneth Moore and Bret Naylor for their technical support in developing the OpenMDAO framework features needed to enable this work and for helping to debug some of the more challenging analytic derivatives. We are also grateful to Chris Snyder for his advice and insight in helping us to understand the CEA tool.

\section{References}

[1] Hooker, J. R., Wick, A., Zeune, C., and Agelastos, A., "Over Wing Nacelle Installations for Improved Energy Efficiency," 31st AIAA Applied Aerodynamics Conference, AIAA Paper 2013-2920, 2013. doi:10.2514/6.2013-2920

-[2] Fujino, M., and Kawamura, Y., "Wave-Drag Characteristics of an Overthe-Wing Nacelle Business-Jet Configuration," Journal of Aircraft, Vol. 40, No. 6, 2003, pp. 1177-1184. doi: $10.2514 / 2.7207$

[3] Smith, A. M. O., and Roberts, H. E., "The Jet Airplane Utilizing Boundary Layer Ingestion for Propulsion," Journal of Aeronautical Sciences, Vol. 14, No. 2, 1947, pp. 97-109.

-[4] Smith, L. H., "Wake Ingestion Propulsion Benefit," Journal of Propulsion and Power, Vol. 9, No. 1, Feb. 1993, pp. 74-82. doi:10.2514/6.1991-2007
[5] Plas, A. P., Sargeant, M. A., Madani, V., Crichton, D., Greitzer, E. M., Hynes, T. P., and Hall, C. A., "Performance of a Boundary Layer Ingesting (BLI) Propulsion System," 45th AIAA Aerospace Sciences Meeting and Exhibit, AIAA Paper 2007-0450, 2007. doi:10.2514/6.2007-450

- [6] Felder, J. L., Kim, H. D., and Brown, G. V., "Turboelectric Distributed Propulsion Engine Cycle Analysis for Hybrid-Wing-Body Aircraft," 47th AIAA Aerospace Sciences Meeting Including The New Horizons Forum and Aerospace Exposition, AIAA Paper 2009-1132, 2009. doi: $10.2514 / 6.2009-1132$.

- [7] Martins, J. R. R. A., and Lambe, A. B., "Multidisciplinary Design Optimization: A Survey of Architectures," AIAA Journal, Vol. 51, No. 9 , Sept. 2013, pp. 2049-2075. doi:10.2514/1.J051895

[8] Drela, M., "Simultaneous Optimization of the Airframe, Powerplant, and Operation of Transport Aircraft," Proceedings of the 2nd Aircraft Structural Design Conference, Royal Aeronautical Soc., London, 2010.

[9] Welstead, J. R., and Felder, J. L., "Conceptual Design of a Single-Aisle Turboelectric Commercial Transport with Fuselage Boundary Layer Ingestion," 54th AIAA Aerospace Sciences Meeting, AIAA Paper 20161027, 2016. doi:10.2514/6.2016-1027

[10] Martins, J. R. R. A., Sturdza, P., and Alonso, J. J., "The Complex-Step Derivative Approximation," ACM Transactions on Mathematical Software, Vol. 29, No. 3, Sept. 2003, pp. 245-262. doi: $10.1145 / 838250.838251$

[11] Martins, J. R. R. A., and Hwang, J. T., "Review and Unification of Methods for Computing Derivatives of Multidisciplinary Computational Models," AIAA Journal, Vol. 51, No. 11, 2013, pp. 2582-2599. doi:10.2514/1.J052184

[12] Nielsen, E. J., and Diskin, B., "Discrete Adjoint-Based Design for Unsteady Turbulent Flows on Dynamic Overset Unstructured Grids," AIAA Journal, Vol. 51, No. 6, 2013, pp. 1355-1373. doi:10.2514/1.J051859

[13] Palacios, F., et al., "Stanford University Unstructured (SU2): OpenSource Analysis and Design Technology For Turbulent Flows," 52nd Aerospace Sciences Meeting, AIAA Paper 2014-0243, 2014. doi: $10.2514 / 6.2014-0243$

[14] Carrier, G., Destarac, D., Dumont, A., Meheut, M., Din, I. S. E., Peter, J., Khelil, S. B., Brezillon, J., and Pestana, M., "Gradient-Based Aerodynamic Optimization with the elsA Software," 52nd Aerospace Sciences Meeting, AIAA Paper 2014-0568, Feb. 2014. doi:10.2514/6.2014-0568

[15] Lyu, Z., Kenway, G. K., Paige, C., and Martins, J. R. R. A., "Automatic Differentiation Adjoint of the Reynolds-Averaged Navier-Stokes Equations with a Turbulence Model," 21st AIAA Computational Fluid Dynamics Conference, AIAA Paper 2013-2581, July 2013. doi: $10.2514 / 6.2013-2581$

[16] Lyu, Z., and Martins, J. R. R. A., "Aerodynamic Design Optimization Studies of a Blended-Wing-Body Aircraft," Journal of Aircraft, Vol. 51, No. 5, Sept. 2014, pp. 1604-1617. doi:10.2514/1.C032491

[17] Lyu, Z., Kenway, G. K., and Martins, J. R. R. A., "Aerodynamic Shape Optimization Investigations of the Common Research Model Wing Benchmark," AIAA Journal, Vol. 53, No. 4, April 2015, pp. $968-985$. doi:10.2514/1.J053318

[18] Chen, S., Lyu, Z., Kenway, G. K. W., and Martins, J. R. R. A., "Aerodynamic Shape Optimization of the Common Research Model Wing-Body-Tail Configuration," Journal of Aircraft, Vol. 53, No. 1, Jan. 2016, pp. 276-293. doi:10.2514/1.C033328

[19] LeDoux, S. T., Vassberg, J. C., Young, D. P., Fugal, S., Kamenetskiy, D., Huffman, W. P., Melvin, R. G., and Smith, M. F., "Study Based on the AIAA Aerodynamic Design Optimization Discussion Group Test Cases," AIAA Journal, Vol. 53, No. 7, 2015, pp. 1910-1935. doi:10.2514/1.j053535

[20] Kenway, G. K. W., and Martins, J. R. R. A., "Multipoint Aerodynamic Shape Optimization Investigations of the Common Research Model Wing," AIAA Journal, Vol. 54, No. 1, Jan. 2016, pp. 113-128. doi:10.2514/1.J054154

[21] Dumont, A., and Meheut, M., "Gradient-Based Optimization of CRM Wing-Alone and Wing-Body-Tail Configurations by RANS Adjoint Technique," 54th AIAA Aerospace Sciences Meeting, AIAA Paper 2016-1293, Jan. 2016. doi: $10.2514 / 6.2016-1293$

[22] Lyu, Z., Xu, Z., and Martins, J. R. R. A., "Benchmarking Optimization Algorithms for Wing Aerodynamic Design Optimization," Proceedings 
of the 8th International Conference on Computational Fluid Dynamics, Paper ICCFD8-2014-0203, Chengdu, Sichuan, PRC, July 2014.

[23] Sanghi, V., Lakshmanan, B. K., and Sundararajan, V., "Survey of Advancements in Jet-Engine Thermodynamic Simulation," Journal of Propulsion and Power, Vol. 16, No. 5, 2000, pp. 797-807. doi: $10.2514 / 2.5644$

[24] Jones, S., "An Introduction to Thermodynamic Performance Analysis of Aircraft Gas Turbine Engine Cycles Using the Numerical Propulsion System Simulation Code," NASA John H. Glenn Research Center TR TM-2007-214690, Cleveland, OH, 2007.

[25] Hendricks, E. S., Jones, S. M., and Gray, J. S., "Design Optimization of a Variable-Speed Power-Turbine," 50th AIAA/ASME/SAE/ASEE Joint Propulsion Conference, AIAA Paper 2014-3445, July 2014. doi: $10.2514 / 6.2014-3445$

[26] Geiselhart, K., Ozoroski, L. P., Fenbert, J. W., Shields, E. W., and Li, W., "Integration of Multifidelity Multidisciplinary Computer Codes for Design and Analysis of Supersonic Aircraft," 49th AIAA Aerospace Sciences Meeting, AIAA Paper 2011-0465, 2011. doi: $10.2514 / 6.2011-465$

[27] Allison, D., Morris, C., Schetz, J., Kapania, R., Sultan, C., Deaton, J., and Grandhi, R., "A Multidisciplinary Design Optimization Framework for Design Studies of an Efficient Supersonic Air Vehicle," 12th AIAA Aviation Technology, Integration, and Operations (ATIO) Conference and 14th AIAA/ISSMO Multidisciplinary Analysis and Optimization Conference, AIAA Paper 2012-5492, 2012. doi:10.2514/6.2012-5492

[28] Allison, D. L., Alyanak, E., and Bhagat, N., "High Fidelity, Nonlinear, Integrated Nozzle Installation Effects for Numerical Propulsion System Simulation," 56th AIAA/ASCE/AHS/ASC Structures, Structural Dynamics, and Materials Conference, AIAA Paper 2015-0649, 2015. doi:10.2514/6.2015-0649

[29] Allison, D. L., Alyanak, E. J., and Shimmin, K., "Aircraft System Affects Including Propulsion and Air Cycle Machine Coupled Interactions," 57th AIAA/ASCE/AHS/ASC Structures, Structural Dynamics, and Materials Conference, AIAA Paper 2016-0671, 2016. doi:10.2514/6.2016-0671

[30] Kenway, G. K. W., Kennedy, G. J., and Martins, J. R. R. A., "Scalable Parallel Approach for High-Fidelity Steady-State Aeroelastic Analysis and Adjoint Derivative Computations," AIAA Journal, Vol. 52, No. 5, 2014, pp. 935-951. doi: $10.2514 / 1 . J 052255$

[31] Hwang, J. T., Lee, D. Y., Cutler, J. W., and Martins, J. R. R. A., "LargeScale Multidisciplinary Optimization of a Small Satellite's Design and Operation," Journal of Spacecraft and Rockets, Vol. 51, No. 5, Sept. 2014, pp. 1648-1663. doi:10.2514/1.A32751

[32] Hwang, J. T., and Martins, J. R. R. A., "A Computational Architecture for Coupling Heterogeneous Numerical Models and Computing Coupled Derivatives," ACM Transactions on Mathematical Software, 2017 (in press).

[33] Tran, D. H., and Snyder, C. A., "Effects of Chemical Equilibrium on Turbine Engine Performance for Various Fuels and Combustor Temperatures," NASA John H. Glenn Research Center TR TM-105399, Cleveland, OH, 1992.

[34] Nuzum, S. R., Donovan, A., Roberts, R. A., and Wolff, M., "Dynamic Modeling at a Vehicle Level of a Cryogenic Based Thermal System for a High Powered System," 57th AIAA/ASCE/AHS/ASC Structures, Structural Dynamics, and Materials Conference, AIAA Paper 20160673, 2016. doi: $10.2514 / 6.2016-0673$

[35] Walter, M. A. T., and Owen, P. R., "Element Potential-Based Procedure for Metamodeling of Combustion Products," Journal of Propulsion and Power, Vol. 30, No. 6, 2014, pp. 1711-1720. doi:10.2514/1.B35189
[36] Gordon, S., and McBride, B. J., "Computer Program for Calculation of Complex Chemical Equilibrium Compositions, Rocket Performance, Incident and Reflected Shocks, and Chapman-Jouguet Detonations," NASA Rept. RP-1311, 1994.

[37] Schecher, W. D., and McAvoy, D. C., "MINEQL+: A Software Environment for Chemical Equilibrium Modeling," Computers, Environment and Urban Systems, Vol. 16, No. 1, 1992, pp. 65-76. doi:10.1016/0198-9715(92)90053-T

[38] Konigsberger, E., and Eriksson, G., "A New Optimization Routine for Chemsage," Calphad, Vol. 19, No. 2, 1995, pp. 207-214. doi:10.1016/0364-5916(95)00021-6

[39] Smith, W. R., and Missen, R. W., Chemical Reaction Equilibrium Analysis: Theory and Algorithms, 1st ed., Wiley, New York, 1982, pp. 140-145.

[40] Goodwin, D. G., Moffat, H. K., and Speth, R. L., "Cantera: An ObjectOriented Software Toolkit for Chemical Kinetics, Thermodynamics, and Transport Processes," Ver. 2.1.2, 2014, http://www.cantera.org/ docs/sphinx/html/faq.html.

[41] Chin, J., Gray, J., Jones, S., and Berton, J., "Open-Source Conceptual Sizing Models for the Hyperloop Passenger Pod," 56th AIAA/ASCE/ AHS/ASC Structures, Structural Dynamics, and Materials Conference, AIAA Paper 2015-1587, 2015. doi:10.2514/6.2015-1587

[42] Haglind, F., and Singh, R., "Design of Aero Gas Turbines Using Hydrogen," Journal of Engineering for Gas Turbines and Power, Vol. 128, No. 4, 2006, pp. 754-764. doi: $10.1115 / 1.2179468$

[43] Fulton, K., "Cryogenic Fueled Turbofans: Kuznetsov Bureau's Pioneer Work on LH2 and LNG Duel-Fuel Engines," Aircraft Engineering and Aerospace Technology, Vol. 65, No. 11, 1993, pp. 8-11. doi:10.1108/eb037431

[44] Leal, A. M., Blunt, M. J., and LaForce, T. C., "A Robust and Efficient Numerical Method for Multiphase Equilibrium Calculations: Application to CO2-Brine-Rock Systems at High Temperatures, Pressures and Salinities," Advances in Water Resources, Vol. 62, Pt. C, Dec. 2013, pp. 409-430. doi:10.1016/j.advwatres.2013.02.006

[45] Lambe, A. B., and Martins, J. R. R. A., "Extensions to the Design Structure Matrix for the Description of Multidisciplinary Design, Analysis, and Optimization Processes," Structural and Multidisciplinary Optimization, Vol. 46, No. 2, 2012, pp. 273-284. doi:10.1007/s00158-012-0763-y

[46] Malcolm, W., Jr., NIST-JANAF Thermochemical Tables, American Inst. of Physics, The American Chemical Soc., 1998.

-[47] Gray, J. S., Hearn, T. A., Moore, K. T., Hwang, J., Martins, J. R. R. A., and Ning, A., "Automatic Evaluation of Multidisciplinary Derivatives Using a Graph-Based Problem Formulation in OpenMDAO," 15th AIAA/ISSMO Multidisciplinary Analysis and Optimization Conference, AIAA Paper 2014-2042, Aug. 2014. doi:10.2514/6.2014-2042

[48] Hwang, J. T., "A Modular Approach to Large-Scale Design Optimization of Aerospace Systems," Ph.D. Dissertation, Univ. of Michigan, Ann Arbor, MI, 2015.

[49] Griewank, A., and Andrea, W., Evaluating Derivatives: Principles and Techniques of Algorithmic Differentiation, SIAM, Philadelphia, 2008.

[50] Martins, J. R. R. A., Alonso, J. J., and Reuther, J. J., "A Coupled-Adjoint Sensitivity Analysis Method for High-Fidelity Aero-Structural Design," Optimization and Engineering, Vol. 6, No. 1, March 2005, pp. 33-62. doi:10.1023/B:OPTE.0000048536.47956.62

[51] Lefebvre, A. H., Gas Turbine Combustion, 3rd ed., CRC Press, Boca Raton, FL, 1998, p. 42.

J. M. Seitzman Associate Editor 
This article has been cited by:

1. Gaetan K. Kenway, Cetin C. Kiris. Aerodynamic Shape Optimization of the STARC-ABL Concept for Minimal Inlet Distortion . [Citation] [PDF] [PDF Plus] 